\title{
Ozonoterapia na Osteoartrose do Joelho: Revisão Sistemática
}

\section{Ozone Therapy in Knee Osteoarthritis: A Systematic Review}

\author{
Tiago COSTA $\triangle^{1,2}$, Santiago RODRIGUES-MANICA ${ }^{1,2}$, Carina LOPES ${ }^{1,2}$, João GOMES ${ }^{1,2}$, José MARONA ${ }^{1,2}$, \\ Sandra FALCÃ $O^{1,2}$, Jaime BRANCO ${ }^{1,2}$ \\ Acta Med Port 2018 Oct;31(10):576-580 • https://doi.org/10.20344/amp.10330
}

\section{RESUMO}

Introdução: O presente estudo teve por objetivo realizar uma revisão sistemática de forma a analisar a eficácia da ozonoterapia na osteoartrose do joelho. Os objetivos visaram avaliar o efeito temporal da ozonoterapia na dor no joelho, na melhoria funcional e na progressão radiográfica.

Material e Métodos: Realizou-se uma pesquisa nas bases de dados PubMed, Embase, Cochrane Library, Scopus e Web of Science a fim de identificar estudos aleatorizados e controlados que tratassem dessa associação. Utilizaram-se os seguintes descritores em língua inglesa: 'ozone therapy', 'knee osteoarthritis'. Realizou-se um resumo descritivo e avaliação de qualidade de todos os estudos incluídos para análise.

Resultados: Identificaram-se seis estudos aleatorizados e controlados relacionados com o objetivo deste trabalho. A avaliação do risco de viés mostrou que um estudo foi considerado como risco moderado de viés e os restantes como risco alto de viés. Não se realizou a análise quantitativa dos dados pois os estudos incluídos não foram suficientemente homogéneos. Os participantes dos estudos eram em geral doentes idosos com osteoartrose do joelho leve a moderada.

Discussão: A variabilidade nas intervenções de ozonoterapia e comparadores, demonstra que não existe uma terapêutica estandardizada. Foram poucos os estudos que relataram os efeitos adversos, e quando aconteceu, estes eram ligeiros e associados ao procedimento.

Conclusão: A ozonoterapia mostrou eficácia a curto prazo, em relação ao placebo e quando combinada com ácido hialurónico, sem ser promissora em relação aos restantes tratamentos vigentes. É importante que novos estudos aleatorizados e controlados avaliem os benefícios/riscos da ozonoterapia tanto a curto como a médio/longo prazo.

Palavras-chave: Osteoartrose do Joelho; Ozono/uso terapêutico

\section{ABSTRACT}

Introduction: The aim of this study was to conduct a systematic review in order to examine the effectiveness of ozone therapy on knee osteoarthritis. The objectives were to evaluate the effect over time of ozone therapy in terms of knee pain, functional improvement and radiographic progression.

Material and Methods: A search was carried out on PubMed, Embase, Cochrane Library, Scopus and Web of Science databases to identify randomized and controlled studies focusing on this association. The following descriptors were used in English: ozone therapy, knee osteoarthritis. A descriptive summary and quality assessment was made of all studies included for analysis.

Results: Six randomized and controlled studies were identified. The risk of bias assessment demonstrated that one study was considered as having a moderate risk of bias and the remainder a high risk of bias. No quantitative analysis of the data was performed, as the studies included were not sufficiently homogeneous. The participants in the studies were generally elderly patients with mild to moderate knee osteoarthritis.

Discussion: The variability of ozone therapy and the comparators demonstrates that there is no standardized therapy. Few studies reported adverse effects, and where they occurred, they were mild and associated with the procedure.

Conclusion: Ozone therapy proved effective in the short-term in relation to placebo and when combined with hyaluronic acid, but it was not superior to other current treatments. More randomised and controlled studies are needed to evaluate the risks/benefits of ozone therapy, both in the short term and the medium/long term.

Keywords: Osteoarthritis, Knee; Ozone/therapeutic use

\section{INTRODUÇÃO}

A osteoartrose do joelho é uma doença osteoarticular que condiciona dor, rigidez e diminuição da função articular. ${ }^{1}$ A elevada prevalência desta doença, especialmente em pessoas idosas, torna-a um dispendioso problema de saúde. ${ }^{2-4} \mathrm{O}$ mecanismo exato da dor ainda não é bem conhecido. A origem da dor tem sido atribuída à cápsula articular, ligamentos, sinovial, osso, menisco, ligamentos e tendões. ${ }^{5,6}$

O tratamento não-cirúrgico destes doentes envolve uma abordagem multimodal que inclui o uso de suplementos com glucosamina/condroitina, fármacos anti-inflamatórios, injeções intra-articulares, acupuntura, proteção articular, fisioterapia, exercícios, ortóteses entre outras. ${ }^{7,8}$ No entanto, nenhuma dessas modalidades alivia completamente a dor no joelho e/ou resolve os restantes sintomas. Num estudo de 2007, nenhum destes tratamentos demonstrou ter uma vantagem significativa sobre os outros. ${ }^{4}$

A ozonoterapia é uma terapêutica que induz efeitos

1. Serviço de Reumatologia. Hospital de Egas Moniz. Centro Hospitalar de Lisboa Ocidental. Lisboa. Portugal.

2. CEDOC. NOVA Medical School/Faculdade de Ciências Médicas. Universidade Nova de Lisboa. Lisboa. Portugal.

$\triangle$ Autor correspondente: Tiago Costa. tiagoafco@hotmail.com

Recebido: 01 de fevereiro de 2018 - Aceite: 19 de Junho de 2018 | Copyright @ Ordem dos Médicos 2018 
anti-inflamatórios e analgésicos e, quando utilizada na concentração terapêutica adequada, pode aliviar a dor e meIhorar a função articular do joelho sem efeitos adversos graves. ${ }^{9} \mathrm{O}$ ozono é também bacteriostático, fungicida e viricida, portanto com risco mínimo de infeção. Ele também estimula o metabolismo do oxigénio e ativa o sistema imunológico. ${ }^{10,11}$

Os benefícios médicos do ozono são cada vez mais conhecidos e colocados em prática nos últimos anos, especialmente para as doenças músculo-esqueléticas, incluindo lombalgia, hérnia discal, doença peri-articular do ombro e osteoartrose do joelho. ${ }^{12}$

Este trabalho visa sistematizar os resultados de estudos controlados e aleatorizados que avaliam a eficácia da ozonoterapia na osteoartrose do joelho.

\section{MATERIAL E MÉTODOS}

Procedeu-se ao registo online desta revisão sistemática no PROSPERO (número de registo: CRD42017080263), sendo realizada segundo as diretrizes da declaração PRISMA.

Critérios de inclusão: estudos controlados e aleatorizados que avaliaram a eficácia da ozonoterapia na osteoartrose do joelho, em humanos. Incluiu-se apenas estudos cujos doentes com osteoartrose do joelho tivessem idade superior ou igual a 18 anos $(P)$. Em todos os estudos teria de existir pelo menos um grupo controlo tratado por agentes além do ozono intra-articular (I), nomeadamente placebo ou outro comparador, fármaco ou não-fármaco (C). Os estudos teriam de ser redigidos em língua inglesa ou portuguesa, no intervalo temporal de 2000 a outubro de 2017 , inclusive.

Critérios de exclusão: Foram excluídos estudos só publicados na forma de abstract.

Objetivos primários e secundários $(\mathrm{O})$ : objetivo primário foi avaliar melhoria da dor com base na escala visual analógica (EVA) ao $1^{\circ}$ mês, $3^{\circ}$ mês, $6^{\circ}$ mês e $12^{\circ}$ mês. Os objetivos secundários foram avaliar a melhoria funcional com base no Western Ontario and McMaster Universities Osteoarthritis Index (WOMAC) funcional ao $1^{\circ}$ mês, $3^{\circ}$ mês, $6^{\circ}$ mês e $12^{\circ}$ mês e avaliar a progressão radiográfica com base na escala de Kellgren-Lawrance ao $6^{\circ}$ mês e $12^{\circ}$ mês. Uma síntese quantitativa será realizada se os estudos incluídos forem suficientemente homogéneos.

Estratégia de pesquisa: dois investigadores realizaram uma pesquisa sistemática no PubMed, Embase, Cochranelibrary, Scopus e Web of Science. A estratégia de pesquisa foi a seguinte: 'ozone therapy'/exp AND 'knee osteoarthritis'/exp AND [2000-2017]/py AND [humans]/lim (Apêndice 1: https://www.actamedicaportuguesa.com/revista/index. php/amp/article/view/10330/Apendice_001.pdf). Dois investigadores reviram todos os títulos e abstracts de forma a remover os duplicados e avaliar a relevância dos mesmos de acordo com os critérios de inclusão e exclusão. Em caso de ambiguidade, realizou-se a revisão de todo o texto. Em caso de discordância, o assunto resolveu-se com recurso a um terceiro investigador.
Extração e análise dos dados: dois revisores realizaram de forma independente a extração de dados usando uma tabela de extração de dados pré-desenvolvida. Extraíram as características basais dos estudos incluídos de modo a formar resumos descritivos. Os dados extraídos foram verificados quanto à consistência, e as discrepâncias foram discutidas até chegar a um consenso. A análise quantitativa dos dados dependerá se os estudos incluídos forem suficientemente homogéneos, relativamente às variáveis descritas nos objetivos.

Avaliação de qualidade: dois investigadores avaliaram de forma independente a qualidade metodológica de cada estudo elegível usando o Cochrane Risk of Bias tool no Review Manager 5.3 para determinar o risco de viés. Avaliaram-se os seguintes domínios: geração da sequência aleatória (viés de seleção), ocultação de alocação (viés de seleção), ocultação de participantes (viés de desempenho), ocultação de profissionais (viés de desempenho), ocultação de avaliadores de desfecho (viés de deteção), desfechos incompletos (viés de atrito), relato seletivo do desfecho (viés de relato), e outras fontes de viés. O risco de viés para cada domínio foi classificado como baixo (+), alto (-) ou incerto (?). ${ }^{13} \mathrm{Um}$ estudo foi considerado como baixo risco de viés apenas quando todos os domínios foram classificados como baixo risco de viés; se um ou dois domínios fossem classificados como risco alto ou moderado de viés, o teste era considerado risco moderado de viés; se mais de dois domínios fossem classificados como risco elevado ou moderado de viés, então considerou-se alto risco de viés. ${ }^{14}$ Em caso de discordância, o assunto foi resolvido com recurso a um terceiro investigador.

\section{RESULTADOS}

No total, incluiu-se na análise seis estudos controlados e aleatorizados, ${ }^{12,15-19}$ publicados entre 2015 e 2017. Os detalhes da pesquisa da literatura estão resumidos num diagrama de fluxo (Fig. 1).

As caraterísticas dos estudos incluídos são apresentadas através de uma tabela descritiva (Apêndice 2: https:// www.actamedicaportuguesa.com/revista/index.php/amp/ article/view/10330/Apendice_002.pdf). Não se realizou a análise quantitativa dos dados pois os estudos incluídos não eram suficientemente homogéneos.

Relativamente às caraterísticas dos doentes, a maioria era do sexo feminino, de idade variável consoante o estudo. Contudo, a maior parte dos estudos incluiu doentes com mais de 50 anos de idade. Apenas três estudos relataram a osteoartrose do joelho como sendo idiopática. ${ }^{15-17}$ Todos os estudos usaram a classificação radiográfica de Kellgren-Lawrance na inclusão de doentes. Destes, quatro estudos apresentavam Kellgren-Lawrance II-III ${ }^{15-17,19}$ e dois estudos apresentavam Kellgren-Lawrance I-II. 12,18 Um estudo $^{19}$ especificou a terapêutica prévia, dois estudos ${ }^{12,18}$ excluíram doentes com toma diária prévia de analgésicos e dois estudos ${ }^{16,17}$ incluíram doentes sem resposta a terapêutica analgésica nos últimos três meses.

Relativamente à intervenção existiram variações entre 


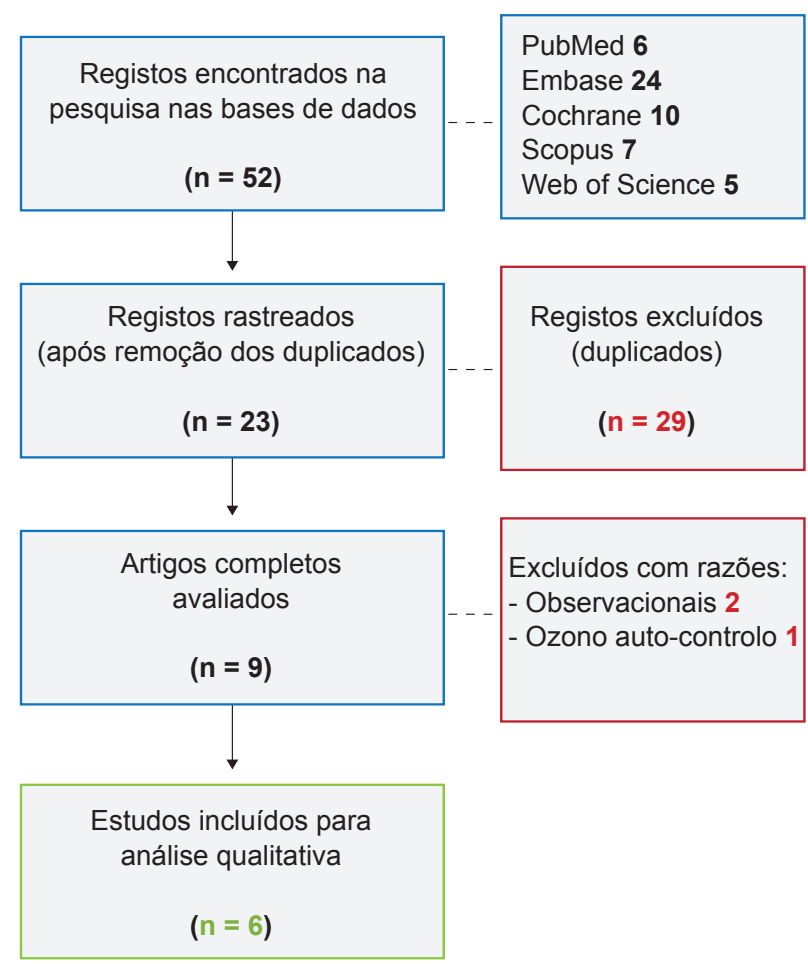

Figura 1 - Diagrama de fluxo do estudo

os estudos: o número de sessões/intervenções variou entre três e doze; a duração do tratamento variou entre três a oito semanas; a quantidade de ozono por injeção variou entre 5 $\mathrm{mL}$ a $20 \mathrm{~mL}$; a concentração de ozono variou entre $15 \mathrm{ug} /$ $\mathrm{mL}$ e $40 \mathrm{ug} / \mathrm{mL}$; a abordagem foi variável sendo a predominante a parapatelar lateral externa/interna.

Os grupos comparadores foram distintos nos diferentes estudos, ao qual incluiu, placebo, ${ }^{19}$ ácido hialurónico, ${ }^{15,16}$ plasma rico em plaquetas, ${ }^{15}$ radiofrequência, ${ }^{17}$ dextrose hipertónica, ${ }^{12}$ celecoxib + glucosamina.$^{18}$

$\mathrm{O}$ desenho do estudo de Jesus et al variou consideravelmente dos restantes pois usou um comparador neutro e ocultação de alocação, participantes e profissionais.

Todos os estudos avaliaram a dor com base na escala visual analógica (EVA). Três estudos ${ }^{12,15,19}$ usaram o WOMAC global mas apenas dois estudos ${ }^{15,19}$ fizeram referência ao WOMAC funcional de forma a avaliar a melhoria funcional. Um estudo ${ }^{16}$ avaliou o Knee injury and Osteoarthritis Outcome Score (KOOS), que também contém uma avaliação funcional. Nenhum estudo avaliou a progressão radiográfica. Os intervalos de seguimento variaram entre os diversos estudos. $\mathrm{O}$ estudo ${ }^{18}$ de menor seguimento foi de seis semanas e o estudo ${ }^{15}$ de maior seguimento foi de 12 meses. Na maioria dos estudos ${ }^{15,16,18,19}$ a durabilidade dos seus efeitos foi avaliado somente a curto prazo (2 - 3 meses após o final do tratamento). A heterogeneidade dos intervalos de avaliação em relação ao EVA encontra-se demonstrada na Tabela 1.

A ozonoterapia apresentou melhores resultados que o grupo placebo na redução da dor e na melhoria da função articular, os mesmos resultados que o grupo da dextrose hipertónica e celecoxib + glucosamina e piores resultados que o grupo da radiofrequência, ácido hialurónico e plasma rico em plaquetas. Por outro lado, a terapêutica combinada de ozono com ácido hialurónico revelou-se eficaz a curto prazo.

Ainda em relação aos comparadores, no estudo com dextrose hipertónica, usou-se as mesmas quantidades de produto e número de sessões em relação à ozonoterapia, obtendo-se resultados semelhantes. Nos estudos que envolveram o uso de radiofrequência, ácido hialurónico e plasma rico em plaquetas, estes tiverem menos número de intervenções em relação à ozonoterapia, obtendo ainda assim melhores resultados.

Apenas dois estudos ${ }^{17,19}$ avaliaram as complicações sendo estas ligeiras e associadas essencialmente à punção. Não foram descritas complicações graves. Nenhum estudo fez referência aos custos associados à terapêutica.

Um resumo da avaliação do risco do viés de todos os estudos incluídos encontra-se ilustrado na Tabela 2. Apenas um estudo ${ }^{19}$ obteve um risco moderado de viés, enquanto os restantes estudos ${ }^{12,15-18}$ obtiveram um alto risco de viés. O suporte para julgamento da avaliação de cada domínio de viés encontra-se descrito no Apêndice 3 (Apêndice 3: https://www.actamedicaportuguesa.com/revista/index.php/amp/article/view/10330/Apendice_003.pdf).

\section{DISCUSSÃO}

Esta revisão sistemática incluiu seis estudos controlados e aleatorizados, sendo todos os estudos recentes, o que demonstra o crescente interesse por esta terapêutica, já com eficácia comprovada em outras aéreas. ${ }^{12}$

O facto de os doentes incluídos nos estudos serem predominantemente do sexo feminino e acima de 50 anos está em harmonia com a prevalência da osteoartrose na

Tabela 1 - Intervalos de avaliação em relação ao EVA

\begin{tabular}{|c|c|c|c|c|c|c|}
\hline $\begin{array}{c}\text { EVA } \\
\text { (do inicio do tratamento) }\end{array}$ & 1 mês & 2 meses & 3 meses & 4 meses & 6 meses & 12 meses \\
\hline Hashemi 2015 & & & & $x$ & & \\
\hline Duymus 2016 & $x$ & & $x$ & & $x$ & $x$ \\
\hline Giombini 2016 & $5 s$ & & $x$ & & & \\
\hline Nabi 2016 & & & & $4 \mathrm{M} 1 \mathrm{~s}$ & & \\
\hline Feng 2017 & $3 s / 6 s$ & & & & & \\
\hline Jesus 2017 & $x$ & $x$ & & $x$ & & \\
\hline
\end{tabular}


Tabela 2 - Avaliação do risco de viés de todos os estudos incluídos
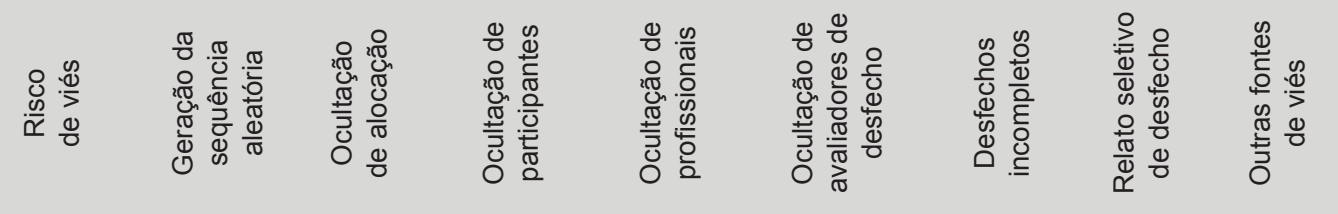

\begin{tabular}{lllllllll}
\hline Hashemi 2015 & Alto & $?$ & $?$ & $?$ & $?$ & $?$ & $?$ & $?$ \\
Duymus 2016 & Alto & + & $?$ & $?$ & $?$ & $?$ & + & + \\
Giombini 2016 & Alto & + & $?$ & $?$ & $?$ & $?$ & $?$ & + \\
Nabi 2016 & Alto & $?$ & $?$ & $?$ & $?$ & $?$ & + & + \\
Feng 2017 & Alto & + & $?$ & $?$ & $?$ & $?$ & $?$ & $?+$ \\
Jesus 2017 & Moderado & + & + & + & + & $?$ & + & + \\
\hline
\end{tabular}

população em geral..$^{2-4}$ As amostras reduzidas $(<100)$ na maior parte dos estudos ${ }^{12,16-19}$ retirou poder estatístico aos resultados obtidos.

Os estudos que não referiram como critério de inclusão a forma de osteoartrose idiopática, podem ter incluído casos de osteoartrose secundária influenciando o resultado final, o que dificulta ainda mais a comparação dos diferentes resultados. Todos os estudos incluíram doentes com osteoartrose ligeira-moderada segundo a classificação radiográfica de Kellgren-Lawrance, deixando de fora os casos de osteoartrose grave o que influenciou positivamente os resultados.

Outra limitação foi a falta de homogeneidade relativamente às terapêuticas prévias ou concomitantes. Alguns estudos referiram como critério de inclusão, a não resposta a terapêutica conservadora prévia, enquanto, outros referiram a exclusão de doentes que realizavam analgésicos diariamente.

A variabilidade nas intervenções (número de sessões, dose/concentração, duração) de ozonoterapia e comparadores, demonstra que não existe uma terapêutica estandardizada. Salienta-se o estudo de Jesus et al que teve a maior duração de tratamento (oito semanas) e o estudo de Feng et al que teve a maior quantidade de ozono por intervenção $(20 \mathrm{~mL})$ e o maior número de intervenções. É razoável que tratamentos mais prolongados e com maior dose produzam melhores resultados e mais duradouros. $\mathrm{O}$ facto de ser um tratamento combinado também deve ser tido em consideração. A abordagem de intervenção é uma das mais frequentemente usadas e permite um fácil acesso intra-articular.

Em relação aos comparadores, destaca-se o estudo de Jesus et al como o único a usar comparador neutro aplicado como procedimento sham. Este foi o único estudo duplo-cego apresentando um risco de viés moderado, enquanto os restantes estudos apresentam um alto risco de viés, o que denota a qualidade dos mesmos.

A grande variação do desenho dos estudos e a presença de comparadores de dimensões variáveis, nomeadamente comprimidos (celecoxib + glucosamina) com injeções intra-articulares, condiciona os resultados obtidos.

O efeito terapêutico do ozono produz significativa re- dução da dor e melhoria funcional de início rápido e com a duração de cerca de três meses. A maioria dos autores recomendam repetir o ciclo de tratamento seis meses depois, sendo que numa frequência pequena de doentes os sintomas podem recidivar após este período de tempo. ${ }^{20}$

Apesar da EVA ser um instrumento validado para aferir a dor, é um parâmetro subjetivo e que pode ser influenciado por outros fatores, nomeadamente sensoriais, emocionais e culturais. Salienta-se ainda o uso de outras escalas, nomeadamente o WOMAC que permite aferir para além da dor e rigidez, a funcionalidade, o que fortifica a qualidade dos estudos que recorreram a este instrumento de avaliação.

O mecanismo de ação envolvido na ozonoterapia envolve a inibição da elevada taxa de óxido nítrico (responsável pela apoptose), inibição de citocinas pró-inflamatórias e melhora da revascularização. ${ }^{21} \mathrm{O}$ estudo de Calunga et al demonstrou que o ozono reduz o stress oxidativo (comprovado por espectrofotometria) e a inflamação (comprovado por ecografia). O stress oxidativo provoca a destruição da cartilagem por degradação direta do colagénio e por ativação das metaloproteinases da matriz e por outro lado, são moléculas de sinalização intracelular que amplificam a resposta inflamatória. ${ }^{22}$ Assim, a maior eficácia da terapêutica combinada de ozono e ácido hialurónico evidenciada no estudo de Giombini et al, pode dever-se ao sinergismo de ambos os mecanismos de ação.

Apesar de serem poucos os estudos que relatam e comentam os efeitos adversos, quando estes existem são ligeiros e de curta duração, o que realça a aparente segurança desta terapêutica a curto prazo. No entanto, é importante referir que o ozono se utiliza como mutagénico em modelos animais e foram encontrados nestes modelos casos de desregulação do ciclo celular por parte de radicais de superóxido. ${ }^{23}$ Assim, desconhece-se a eventual ocorrência de efeitos adversos graves a médio e longo prazo.

\section{CONCLUSÃO}

A ozonoterapia no tratamento da osteoartrose do joeIho mostrou eficácia a curto prazo, em relação ao placebo e quando combinada com ácido hialurónico, sem, no entanto, demonstrar ser superior em relação aos restantes 
tratamentos vigentes (relativamente à EVA e/ou WOMAC). Atualmente, não existem provas convincentes a favor de uma relação benefício/risco positiva da ozonoterapia na osteoartrose do joelho. Assim, é necessário investigar o efeito do ozono na osteoartrose do joelho em ensaios controlados e aleatorizados, duplamente cegos, alargados e homogéneos em que usem comparadores adequados, garantindo sempre que os doentes em ambos os grupos tenham um estado clínico basal semelhante. Relativamente aos outcomes, é importante garantir uma certa homogeneidade, através de escalas validadas. Apesar da elevada subjetividade das escalas atualmente validadas, será importante recorrer ao EVA da dor ou à escala WOMAC. É ainda fundamental avaliar o dano estrutural e progressão da osteoartrose do joelho com exames como radiografia ou a ressonância magnética. Por outro lado, também é importante um seguimento dos doentes tanto a curto como médio/longo prazo, com avaliação dos efeitos adversos e custos.

\section{REFERÊNCIAS}

1. Hawamdeh ZM, Al-Ajlouni JM. The clinical pattern of knee osteoarthritis in Jordan: a hospital based study. Int J Med Sci. 2013;10:790-5.

2. Gupta S, Hawker GA, Laporte A, Croxford R, Coyte PC. The economic burden of disabling hip and knee osteoarthritis (OA) from the perspective of individuals living with this condition. Rheumatology. 2005;44:1531-7.

3. Li Y, Wei X, Zhou J, Wei L. The age-related changes in cartilage and osteoarthritis. Biomed Res Int. 2013:916530.

4. Samson DJ, Grant MD, Ratko TA, Bonnell CJ, Ziegler KM, Aronson N. Treatment of primary and secondary osteoarthritis of the knee. Evid Rep Technol Assess. 2007:1-157.

5. Peat G, McCarney R, Croft P. Knee pain and osteoarthritis in older adults: a review of community burden and current use of primary health care. Ann Rheum Dis. 2001;60:91-7.

6. Felson DT. The sources of pain in knee osteoarthritis. Curr Opin Rheumatol. 2005;17:624-8.

7. Shengelia R, Parker SJ, Ballin M, George T, Reid MC. Complementary therapies for osteoarthritis: are they effective? Pain Manag Nurs. 2013;14:e274-88.

8. Buckwalter JA, Stanish WD, Rosier RN, Schenck RJ, Dennis DA, Coutts $\mathrm{RD}$. The increasing need for nonoperative treatment of patients with osteoarthritis. Clin Orthop Relat Res. 2001:36-45.

9. Mishra SK, Pramanik R, Das P, Das PP, Palit AK, Roy J, et al. Role of intra-articular ozone in osteo-arthritis of knee for functional and symptomatic improvement. Ind J Phys Med Rehabilit. 2011;22:65-9.

10. Maslennikov OV, Kontorshchikov CN, Gribkova IA. Ozone therapy in practice. Health Manual. Nizhny Novgorod Russia. 2008:6-12.

11. Elvis A, Ekta J. Ozone therapy: a clinical review. J Nat Sci Biol Med. 2011;2:66-70.

12. Hashemi M, Jalili P, Mennati S, Koosha A, Rohanifar R, Madadi F, et al. The effects of prolotherapy with hypertonic dextrose versus prolozone (intraarticular ozone) in patients with knee osteoarthritis. Anesth Pain Med. 2015;5:e27585

13. Higgins JT, Green S. Cochrane handbook for systematic reviews of interventions version 5.1.0 [updated March 2011]: The Cochrane Collaboration; 2011.

\section{PROTECÇÃO DE PESSOAS E ANIMAIS}

Os autores declaram que os procedimentos seguidos estavam de acordo com os regulamentos estabelecidos pelos responsáveis da Comissão de Investigação Clínica e Ética e de acordo com a Declaração de Helsínquia da Associação Médica Mundial.

\section{CONFIDENCIALIDADE DOS DADOS}

Os autores declaram ter seguido os protocolos do seu centro de trabalho acerca da publicação de dados. Consentimento do doente obtido.

\section{CONFLITOS DE INTERESSE}

Os autores declaram não terem qualquer conflito de interesse relativamente ao presente artigo.

\section{FONTES DE FINANCIAMENTO}

Os autores declaram não ter recebido subsídios ou bolsas para a elaboração do artigo.

14. Laudy AB, Bakker EW, Rekers M, Moen MH. Efficacy of platelet-rich plasma injections in osteoarthritis of the knee: a systematic review and metaanalysis. Br J Sports Med. 2015;49:657-72.

15. Duymus T, Mutlu S, Dernek B, Komur B, Aydogmus S, Kesiktas F. Choice of intra-articular injection in treatment of knee osteoarthritis: platelet-rich plasma, hyaluronic acid or ozone options. Knee Surg Sports Traumatol Arthrosc. 2017;25:485-92.

16. Giombini A, Menotti F, Di Cesare A, Giovannangeli F, Rizzo M, Moffa $\mathrm{F}$, et al. Comparison between intrarticular injection of hyaluronic acid, oxygen ozone, and the combination of both in the treatment of knee osteoarthrosis. J Biol Regul Homeost Agents. 2016;30:621-5.

17. Nabi NB, Sediginegad A, Haghighi M, Mohamad Zadeh A, Biazar G, Khoshrang $\mathrm{H}$. A comparison of two treatment methods of radiofrequency \& intra-peri articular ozone injection in the improvement of knee osteoarthritis. Pain practice. Int J Med Res Health Sci. 2016,5:539-46.

18. Feng $X$, Beiping $L$. Therapeutic efficacy of ozone injection into the knee for the osteoarthritis patient along with oral celecoxib and glucosamine. $\mathrm{J}$ Clin Diagn Res. 2017;11:UC01-3.

19. Lopes de Jesus CC, Dos Santos FC, de Jesus LM, Monteiro I, Sant'Ana MSSC, Trevisani VF. Comparison between intra-articular ozone and placebo in the treatment of knee osteoarthritis: a randomized, doubleblinded, placebo-controlled study. PLoS One. 2017;12:e0179185.

20. Bernal DS, Rovira-Duplaa G, Monerris-Tabasco M, Gonzalez-Palomares M, Mazo-Sanchez V. Tratamiento de la gonalgia por gonartrosis con ozono intraarticular. Rev Soc Esp Dolor. 2013;20:107-12.

21. Milanes JR, Acosta W, Trinchet E. La ozonoterapia/resultados del tratamiento en pacientes con artrosis degenerativa sintomática de rodilla. Revista Mexicana de Algologia; 2009;6:18-23.

22. Calunga JL, Menéndez S, León R, Chang S, Guanche D, Balbin A, et al. Application of ozone therapy in patients with knee osteoarthritis. Ozone: Science and Engineering. 2012. 34:469-475.

23. Foksinski M, Bilkowski K, Skiba M, Ponikowska I, Szmurlo W, Olinski R. Evaluation of 8-oxodeoxyguanosine, typical oxidative DNA damage, in lymphocytes of ozone-treated arterioscleotic patients. Mutat Res. 1999;438:23-7. 